\title{
Unsteady Flows Characteristics in a Channel with Oblique Plates
}

\author{
Yinxiao Zhan, Tae Seon Park \\ School of Mechanical Engineering, Kyungpook National University, Daegu, South Korea \\ Email: tsparkjp@knu.ac.kr
}

Received 30 April 2015; accepted 19 August 2015; published 26 August 2015

\begin{abstract}
Unsteady flows in a channel with oblique plates have been numerically investigated. The oblique plates as disturbance promoters are installed at the upper wall. Unsteady characteristics are examined for $\operatorname{Re}=350$ and the plate angles of $\alpha=60^{\circ}-120^{\circ}$. The flow fields represent three-dimensional features variously as the plate angle varies. From frequency analysis, it is noted that the disturbed flow by the oblique plates has peculiar unsteady modes. As the flow is more unstable, multiple frequencies are appeared.
\end{abstract}

\section{Keywords}

Unsteady Flow, Frequency, Oblique Plate

\section{Introduction}

Many researchers have paid much attention to the unsteady flow of a channel having various obstacles such as fins, ribs, baffles or plates [1]-[10]. The highly unsteady flows have positive influences on the heat transfer enhancement, because it can provoke the flow mixing [1] [2]. In particular, the plate of those obstacles has the geometric advantages to control the flow unsteadiness depending on the variation of plate height, plate interval, and inclination angle. In general, the laminar flow in a channel becomes turbulent flows, as the Reynolds number increases. The turbulent flow is characterized by chaotic changes related to coherent vortices of many scales. So, the occurrence of many vortices can be connected directly to the turbulent flow or unsteady laminar flow. From this point of view, the disturbed flow by the plate is very interesting to study the unsteady evolution.

From the previous studies [3] [4] for a channel having the plates/baffles mounted normally in the streamwise direction on both channel walls, it was found that the flow instability is strongly correlated to the wall heat transfer. Zhan and Park [5] studied the effect of insulated oblique plates on oscillating flow in a channel. The result revealed the significant heat transfer enhancement by activating unsteady flow due to the oblique plate mounted on the channel wall. Yang et al. [6]-[8] identified that the velocity fluctuation is responsible for the heat transfer enhancement. They investigated the effects of baffle interval and Reynolds number on the flow instabilities. On the other hand, several researchers [9] [10] investigated the influences of baffle shape on the heat transfer in a duct flow. The results showed that the vortical flows caused by the angled baffles induce the increase in heat transfer rate. Accordingly, we can expect that the development of unsteady vertical flows is a 
primary factor to enhance the heat transfer in a channel or duct. However, when obstacles are introduced in a channel or duct, the detailed evolution of unsteady flows is scarcely reported in the literature.

In the present study, the unsteady flow for the channel having a wall with periodic plates is investigated. For a channel with various obstacles, as the Reynolds number increases, the two-dimensional steady flow becomes a time-periodic and chaotic flow. This can be explained by a bifurcation theory. An example is Hopf bifurcation where a steady state bifurcates into a time-periodic state. Compared with other obstacles, the plate has a geometrical advantage to realize various flow conditions using one configuration. The channel flow with plates can be diversely changed depending on the inclination of plates. So, this study attempts a parametric study to examine the effects of geometrical variation on the unsteady flow characteristics for the channel flow with plates. The plate interval is $2 \mathrm{H}$ and the plate length is $0.4 \mathrm{H}$. The inclination angle of the plates are changed for $\alpha=60^{\circ}-120^{\circ}$. The effects of the inclination angle on the critical Reynolds number for Hopf bifurcation and the spectral analysis are discussed. After that, as the two-dimensional unsteady flow develops further, the flow transits to three-dimensional structure. So, several simulations are performed to elucidate three-dimensional unsteady flow characteristics

\section{Numerical Methods and Flow Condition}

\subsection{Governing Equation}

The flow is two-dimensional with constant properties. The $\mathrm{x}$-axis is taken in the flow direction and the $y$-axis is perpendicular to the flow direction. The flow is assumed to be laminar and incompressible. Buoyancy induced effects are neglected. The governing equations for continuity, momentum and temperature are given by

$$
\begin{gathered}
\frac{\partial}{\partial x_{i}}\left(\rho U_{i}\right)=0 \\
\frac{\partial U_{i}}{\partial t}+\frac{\partial}{\partial x_{j}}\left(\rho U_{i} U_{j}\right)=-\frac{\partial P}{\partial x_{j}}+\frac{\partial}{\partial x_{j}}\left[\mu\left(\frac{\partial U_{i}}{\partial x_{j}}+\frac{\partial U_{j}}{\partial x_{i}}\right)\right]+F_{1} \delta_{1 i}
\end{gathered}
$$

where $U_{i}, P, \mu$, are the velocity components, pressure, temperature, viscosity and Prandtl number, respectively. Quantities such as $\rho, \mu$ are set as air properties at room temperature. $F_{1}$ represent the stream wise mean pressure gradient, which needs to be calculated dynamically in order to maintain a constant mass flow rate and $\delta_{1 i}$ is the kronecker delta.

\subsection{Numerical Methods and Boundary Condition}

The spatial discretization is performed with the fourth-order compact scheme. The viscous term and the other terms are evaluated by the fourth-order central difference. A nonstaggered grid arrangement is adopted and the momentum interpolation techniqueis employed to avoid pressure-velocity decoupling. The PISO algorithm is employed for pressure-velocity coupling [11]. The momentum and energy equations are solved with secondorder upwind scheme. Zhan and Park [5] adopted the same computational method. Periodic boundary condition with constant mass flow rates is specified at the inlet and outlet of the channel. No-slip and constant wall temperature conditions are specified on the upper and lower walls of the channel. Oblique plate is insulated.

Figure 1 shows the flow geometry and the coordinate system. It is consisted of two walls channel of height $\mathrm{H}$ with insulated a thin oblique plate $(t=0.02 \mathrm{H})$ on the upper wall. No-slip and constant wall temperature

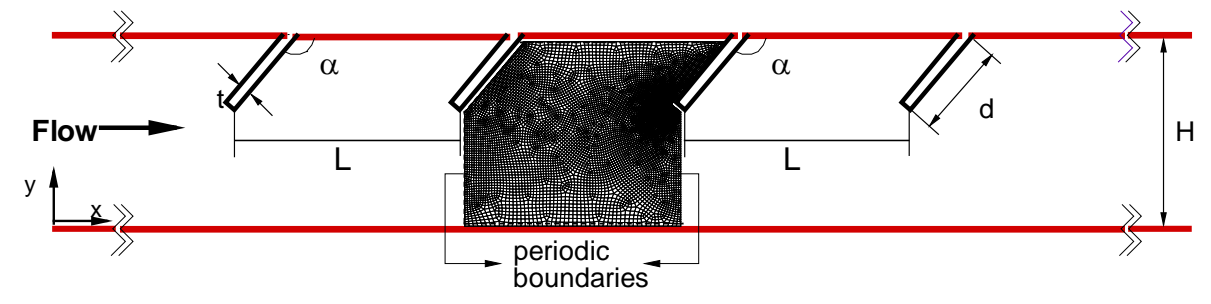

Figure 1. Flow configuration. 
conditions are specified on the upper and lower walls of the channel. The lengths of the oblique plates $d$ is $0.4 \mathrm{H}$. Periodicity lengths $L$ is fixed at $2 H$. For these cases, the inclination angle of the plates limited in $\alpha=60^{\circ}$ $120^{\circ}$ is defined as $90^{\circ} \pm n 10^{\circ}(n=1,2,3,4)$. Oblique plate is insulated. Because the flow is maintained at $\mathrm{Re}=$ 350 , the steady flow can be bifurcated by the variation of tilt angles.

\section{Result and Discussion}

In order to examine the three-dimensional heat transfer for channel with oblique plates, two-dimensional simulations is performed to analyze basic transition process from the steady state to unstable state. For all selected tilt angle, the relationship between the flow frequency and the tilt angle is studied.

\subsection{Two-Dimensional Flows}

The presence of periodic oblique plates in a channel wall makes the flow unstable. When the flow evolves from a steady state to time-dependent self-sustained periodic flows, the critical Reynolds number $\left(\operatorname{Re}_{c r}\right)$ can be defined. As the tilt angle changes, $\mathrm{Re}_{c r}$ is significantly changed. Because the different $\alpha$ gives different unsteady flows.

Figure 2 demonstrates the time history of the $u$-velocity at $x=L / 5, \quad y=H-d \cos \alpha$ for $\operatorname{Re}=350$. As can be seen that for the predicted flows are unsteady for all tilt angles, and oscillation amplitude is increased and then decreased as the tilt angle increases. The highest of flow oscillation amplitude is also observed for $100^{\circ}$, and it is obviously larger for $\alpha=90^{\circ}+n 10^{\circ}$ than that for $\alpha=90^{\circ}-n 10^{\circ}$.

In order to determine the unsteady flow characteristics, i.e., main fundamental frequencies and harmonics. As the tilt angle increases, Fourier power spectra of $u$-velocity and $u$-and $v$-phase portrait for $\alpha=60^{\circ}-120^{\circ}$, at $\operatorname{Re}=$ 350 during $10 \mathrm{~T}$ are showed in Figure 3. One can see that flow have different frequency characteristic for each tilt angle. When $\alpha=60^{\circ}$, flow bifurcate into time-dependent periodic flow characterized by one fundamental frequency $f_{1}$. The phase space trajectory approaches a limit cycle. When $\alpha=70^{\circ}$, flow with one frequency $f_{1}$ and harmonics $2 f_{1}$, its trajectory of $u$ and $v$ velocity is limit cycle also. As increasing tilt angle to $80^{\circ}$, a secondary instability occurs and a new characteristic fundamental frequency $f_{2}$ and their linear combinations $\left(m f_{1}+n f_{2}\right)$ appears, which represents the flow evolved into a quasi periodic flow regime [12]. Here $m$ and $n$ are integers. As we conjectured, the tilt angles of the plate completely control the flow field. The flow with large-amplitude oscillations is more unstable and is more easily to bifurcate into chaotic regime.
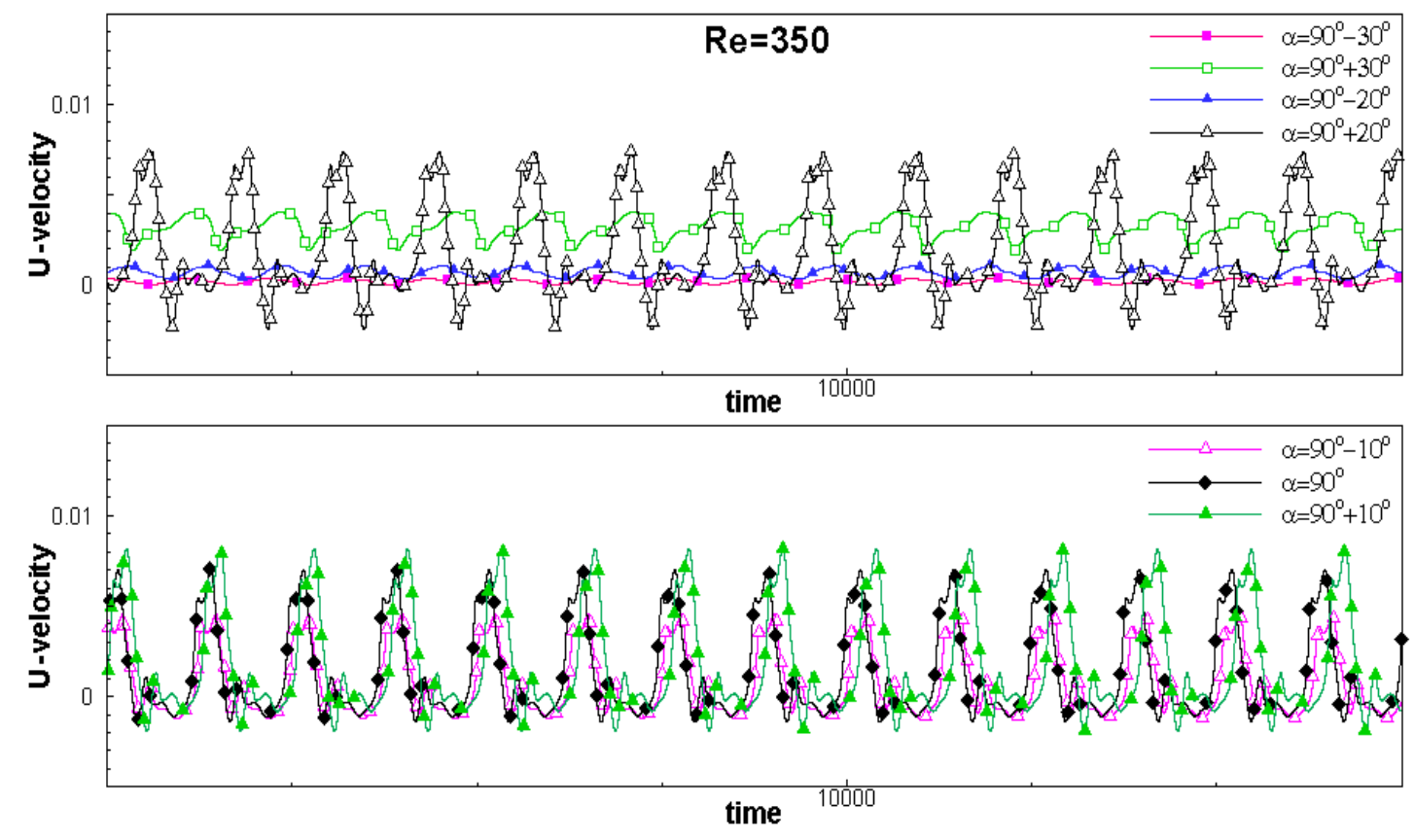

Figure 2. Time history of the $\mathrm{U}$ velocity at $x=L / 5, \quad y=H-d \cos \alpha$, for $\operatorname{Re}=350$. 

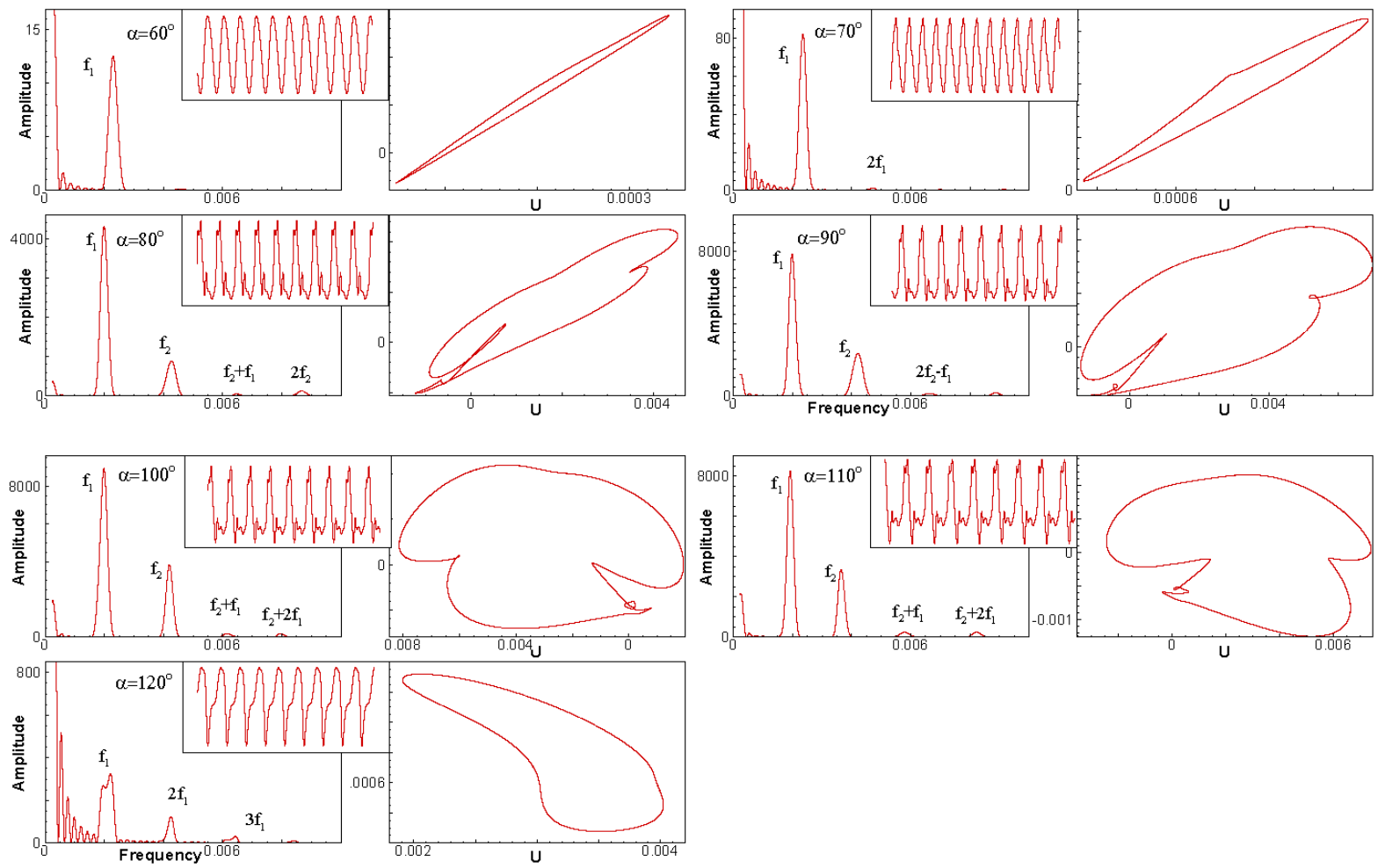

Figure 3. Fourier power spectra and phase portraits of u-and v-velocity at $\mathrm{Re}=350$.

\subsection{Characteristics of Three-Dimensional Flows}

According to the above analysis, the change of the tilt angle brings significant change in flow characteristic. In order to detect the 3D flow, the two-dimensional flow field is expanded in the spanwise direction. Figure 4 shows the time evolution of u-velocity for all tilt angles at two different points. Both of two points are taken at $x=L / 5, y=H-d \cos \alpha$, and in span wise direction, $z=0, H / 2$ respectively. For all tilt angles, two-dimensional T-S wave grows with time and becomes unstable to three-dimensional perturbation. The two curves are identical before the appearance of the 3D disturbance, due to the secondary instability which induces the three-dimensional flow is not prevailing compared with the basic flow. After the different initial time, however, the secondary instability becomes big enough to distort the basic flow, the two curves deviate both in magnitude and in phase. 3D perturbation is infinitesimal for $\alpha=60^{\circ}$. For different tilt angle, initial time that the base mean flow is distorted leading to 3D flow is different. When $\alpha=90^{\circ}$, the initial time is shortest. That may be influence the heat transfer in 3D. Figure 5 shows instantaneous streamlines of upper wall for $\alpha=60^{\circ}-120^{\circ}$, for $\operatorname{Re}=$ $350, t=4457.862$. The parallel streamlines are obtained for $\alpha=60^{\circ}$. This confirm that the mean flow have not distorted yet. For the other tilt angle, the flow field is clearly bifurcated to three dimensional. And one can notice that the flow is more distorted near the oblique plates. Corresponding to the frequency characteristic (Figure 3), as the tilt angle increases, vortex is expanded to spanwise direction, inducing the appearance of the multiple frequencies.

\section{Conclusions}

Unsteady flow and heat transfer in a channel with oblique plates have been numerically investigated. The inclination angle of the plates is changed in a range of $\alpha=60^{\circ}-120^{\circ}$. Flow characteristics are investigated for $\operatorname{Re}=350$.

As the tilt angle changes, the flows evolve from steady state to periodic state with one primary frequency and then to quasi periodic with two primary frequencies and their linear combinations $\left(m f_{1}+n f_{2}\right)$. In particularly, two-dimensional flow with multiple frequencies can be easily three-dimensional. For the range of $\alpha=90^{\circ}-110^{\circ}$, 

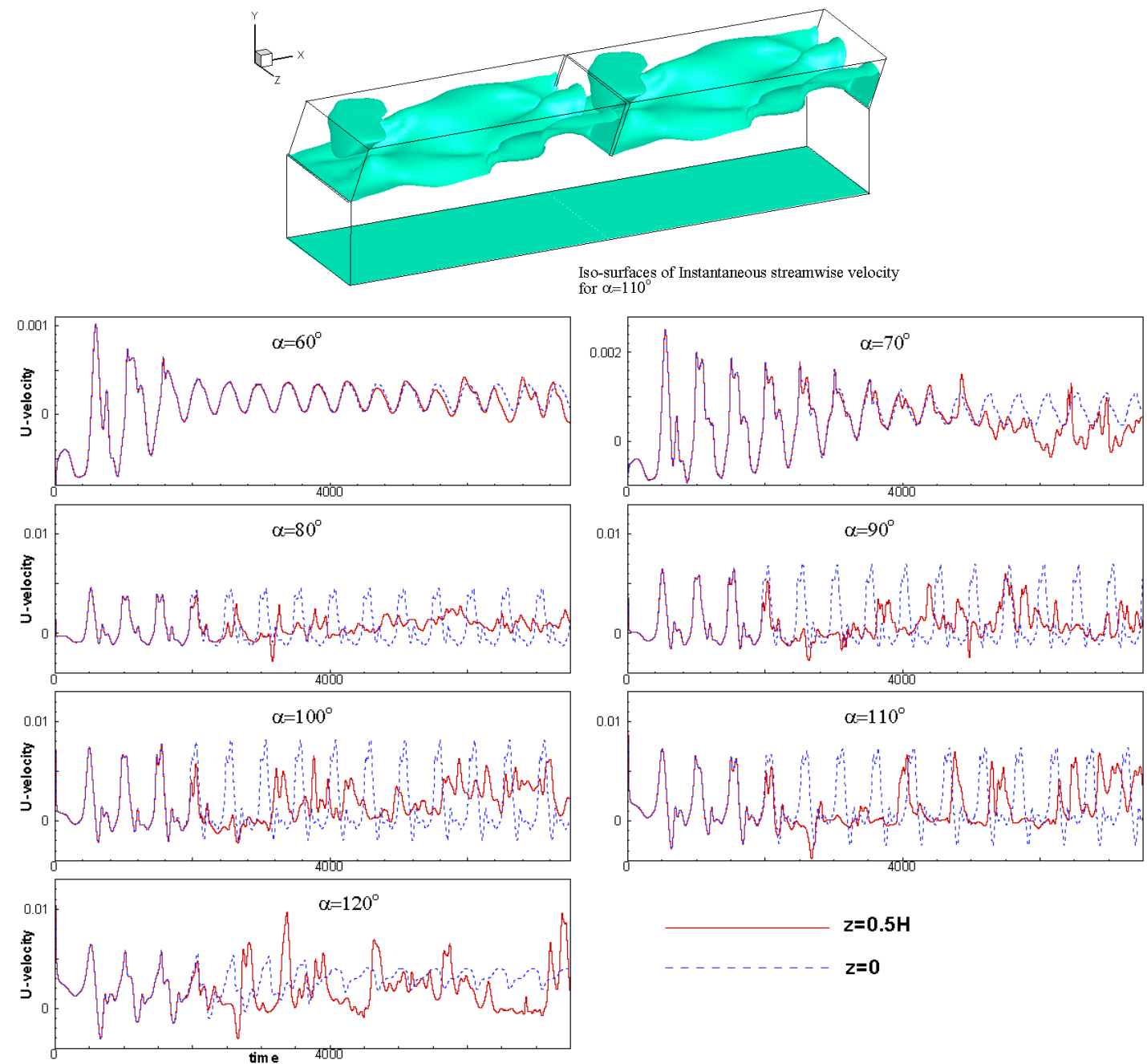

$\mathrm{z}=0.5 \mathrm{H}$

$z=0$

Figure 4. Three-dimensional geometry and time histories of $u$ at two spanwise location: $\mathrm{Re}=350$.
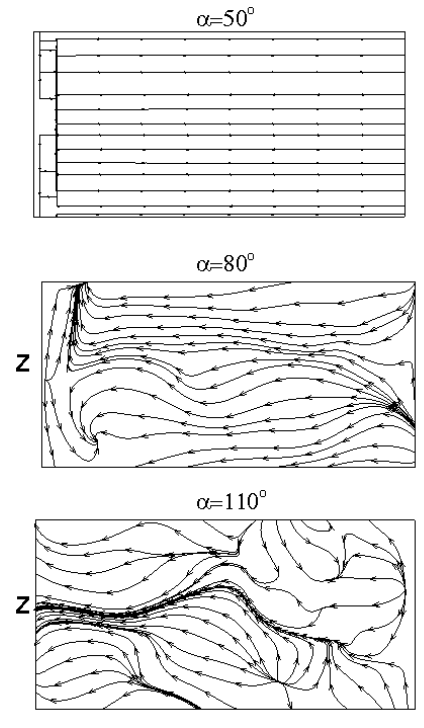

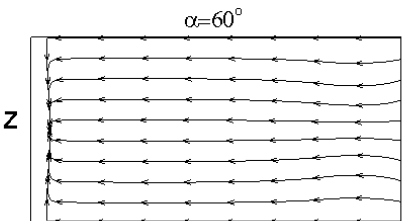

$\alpha=90^{\circ}$

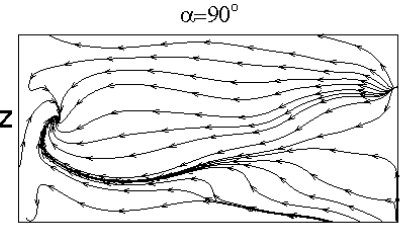

$\alpha=120^{\circ}$

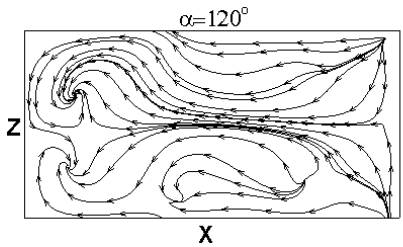

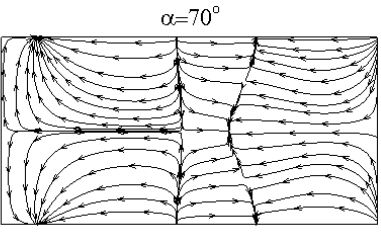

$\alpha=100^{\circ}$
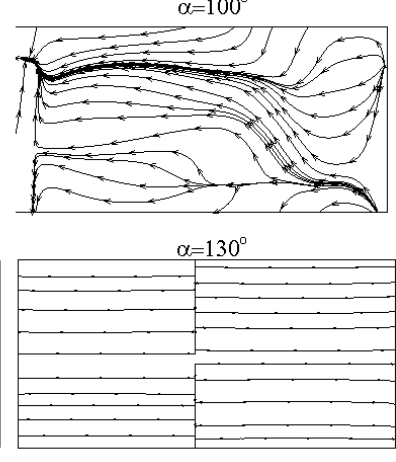

Figure 5. Instantaneous streamlines for $\alpha=60^{\circ} \sim 120^{\circ}, \operatorname{Re}=350(\mathrm{y}=0.95 \mathrm{H})$. 
the transition from the periodic state to chaotic state is obtained quickly. For the geometric condition having multiple frequencies, the flow becomes chaotic not turbulent, because coherent vortices are not produced.

\section{References}

[1] Rowley, G.J. and Patankar, S.V. (1983) Analysis of Laminar Flow and Heat Transfer in Tubes with Internal Circumferential Fins. Int. J. Heat Mass Transfer, 27, 553-560. http://dx.doi.org/10.1016/0017-9310(84)90028-0

[2] Mackley, M.R. and Stonestreet, P. (1995) Heat Transfer and Associated Energy Dissipation for Oscillatory Flow in Baffled Tubes. Chemical Engineering Science, 50, 2211-2224. http://dx.doi.org/10.1016/0009-2509(95)00088-M

[3] Cheng, C.H. and Huang, W.H. (1991) Numerical Prediction for Laminar Forced Convection in Parallel-Plate Channels with Transverse Fin Arrays. Int. J. Heat Mass Transfer, 34, 2739-2749. http://dx.doi.org/10.1016/0017-9310(91)90232-4

[4] Korichi, A., Oufer, L. and Polidori, G. (2009) Heat Transfer Enhancement in Self-Sustained Oscillatory Flow in a Grooved Channel with Oblique Plates. Int. J. Heat Mass Transfer, 52, 1138-1148. http://dx.doi.org/10.1016/j.ijheatmasstransfer.2008.09.013

[5] Zhan, Y.X. and Park, T.S. (2014) Effect of Insulated Oblique Plates on Heat Transfer and Recirculating Flow in a Channel. Journal of Applied Mathematics and Physics, 2, 405-410. http://dx.doi.org/10.4236/jamp.2014.26048

[6] Yang, K.S. (2000) Numerical Investigation of Instability and Transition in an Obsturcted Channel Flow. AIAA Journal, 38, 1173-1178. http://dx.doi.org/10.2514/2.1111

[7] Kang, C.W. and Yang, K.S. (2011) Heat Transfer Characteristics of Baffled Channel Flow. Journal of Heat Transfer, 133, Article ID: 091901-1. http://dx.doi.org/10.1115/1.4003829

[8] Kang, C.W. and Yang, K.S. (2012) Flow Instability in Baffled Channel Flow. Int. J. Heat and Fluid Flow, 38, 40-49. http://dx.doi.org/10.1016/j.ijheatfluidflow.2012.08.002

[9] Kwankaomeng, S. and Promvonge, P. (2010) Numerical Prediction on Laminar Heat Transfer in Square Duct with $30^{\circ}$ Angled Baffle on One Wall. International Communications in Heat and Mass Transfer, 37, 857-866. http://dx.doi.org/10.1016/j.icheatmasstransfer.2010.05.005

[10] Promvonge, P., Jedsadaratanachai, W., Kwankanomeng, S. and Thianpong, C. (2012) 3D Simulation of Laminar Flow and Heat Transfer in V-Baffled Square Channel. International Communications in Heat and Mass Transfer, 39, 85-93. http://dx.doi.org/10.1016/j.icheatmasstransfer.2011.09.004

[11] Park, T.S. (2006) Effects of Time-Integration Method in a Large-Eddy Simulation Using The PISO Algorithm: Part I-Flow Field. Numerical Heat Transfer, Part A, 50, 229-245. http://dx.doi.org/10.1080/10407780600602374

[12] Guzmán, A.M. and Amon, C.H. (1994) Transition to Chaos in Converging-Diverging Channel Flows: Ruelle-TakensNewhouse scenario. Phy. Fluids, 6, 1994-2002. http://dx.doi.org/10.1063/1.868206 